\title{
Unilateral Lesions of the Dorsal Striatum in Rats Disrupt Responding in Egocentric Space
}

\author{
Peter J. Brasted, ${ }^{1}$ Trevor Humby, ${ }^{1}$ Stephen B. Dunnett, ${ }^{1,2}$ and Trevor W. Robbins ${ }^{1,2}$ \\ ${ }^{1}$ Medical Research Council Cambridge Centre for Brain Repair and ${ }^{2}$ Department of Experimental Psychology, University \\ of Cambridge, Cambridge, CB2 2PY, United Kingdom
}

Rats were trained in a specially designed, multichoice operant chamber on a visual choice reaction time task designed to assess performance on each side of the rat's body. The task required animals to sustain a nose poke in a central hole, until a brief light stimulus was presented in either of two holes that were located on the same side of the box. Once the rats were trained to perform the task to both sides independently they received unilateral injections of quinolinic acid into the dorsal striatum.

Postoperatively, lesioned animals were impaired when performing the task on the side contralateral to the lesion. The time taken to initiate contralateral responses was increased. Con- tralateral responses were also exclusively biased toward the nearer of the two response locations, regardless of the location of the stimulus. This was interpreted as a specific impairment in generating responses in contralateral space. In contrast, no comparable deficit was seen when the animals performed the task on the side ipsilateral to the lesion. Additional postoperative challenges, in which response options were presented bilaterally, showed this response deficit to be defined in egocentric coordinates, with the severest response deficits for the most contralateral locations.

Key words: striatum; neglect; rat; excitotoxin; movement; egocentric; Huntington's disease
Unilateral lesions of the dorsal striatum in rats impair responses to contralateral stimuli (Mittleman et al., 1988, Brown and Robbins 1989b, Mayer et al., 1992). Furthermore, because these studies dissociated the locations of the cued response from the cue itself, this "striatal neglect" is believed to reflect the specific inability to produce a motor response to the side contralateral to the lesion. This is consistent with movement disorders that involve striatal degeneration, such as Huntington's disease (Harper, 1996).

Different manifestations of neglect have been shown both experimentally and clinically. As well as an attentional or representational neglect (Bisiach and Luzzatti, 1978; Bisiach et al., 1979; Posner et al., 1984), an "intentional" or output neglect has been demonstrated after damage to anterior cortical or basal ganglia structures, which is characterized by a hemispatial hypokinesia (Watson et al., 1978; Heilman et al., 1985; Tegner and Levander, 1991). The contralateral deficit can also be defined in various coordinate systems. Patients can exhibit a viewer-centered neglect, as defined by the subjects' midline (Farah et al., 1990; Karnath et al., 1991), or an object- or environment-centered neglect, which is defined by external referents (Ladavas 1987; Caramazza and Hills, 1990; Driver and Halligan, 1991). It has been suggested, however, that not all aspects of contralateral space are necessarily affected. For example, subjects can show neglect in immediately adjacent (peripersonal) space but operate normally in far, extrapersonal space (Bisiach et al., 1986; Halligan and Marshall 1991). The reverse pattern of deficits has also been shown (Shelton et al., 1990; Cowey et al., 1994).

In a detailed analysis of rats with unilateral striatal dopamine

Received Aug. 6, 1997; accepted Sept. 5, 1997.

This work was supported by the Medical Research Council (United Kingdom) We thank E. Torres for assistance with the histological analysis.

Correspondence should be addressed to Peter J. Brasted, Medical Research Council Cambridge Centre for Brain Repair, University of Cambridge, Forvie Site, Robinson Way, Cambridge, CB2 2PY, UK.

Copyright (C) 1997 Society for Neuroscience $\quad 0270-6474 / 97 / 178919-08 \$ 05.00 / 0$ depletion, Brown and Robbins (1989a) designed a visual reaction time paradigm to determine whether the impairment in initiating contralateral movement (Dunnett and Björklund, 1983; Carli et al., 1985, 1989) reflected a deficit that was defined in egocentric or allocentric (i.e., externally based) coordinates. The authors demonstrated that animals with dopamine-depleting lesions in the contralateral striatum biased their responding toward the nearer of two response locations, whereas ipsilaterally lesioned animals were unaffected in their responding. This was interpreted as a disruption of egocentrically organized response space.

The neglect of contralateral space after unilateral excitotoxic lesions of intrinsic striatal neurons has yet to be fully quantified. This study sought to address this, with a paradigm that required animals to respond in one of two locations that were both located in the same spatial hemifield (as in the study by Brown and Robbins 1989a). However, in this study, animals were trained to perform the task to both sides of space, separately. This approach has two advantages. First, it provides a comparison between the two sides within the same group of animals. Second, it allows any postoperative impairment to be probed with bilaterally presented response options. It is thus possible to ascertain whether neglect after unilateral striatal lesions is spatially uniform, or whether it reflects an inability to respond to relatively contralateral locations.

\section{MATERIALS AND METHODS}

\section{Subjects}

Eighteen male Lister Hooded rats (Charles River, Cambridge, UK) were used in this experiment. They were housed in groups of two to four. They were kept on a $12 \mathrm{hr}$ light/dark cycle (lights on 9.30 P.M.), and were run in the dark half of the cycle. Their food intake was restricted to 15-17 gm of laboratory chow at the end of each day to maintain $\sim 90 \%$ of free-feeding body weight. Water was available ad libitum. Subjects weighed $\sim 220 \mathrm{gm}$ at the start of testing and weighed $350-450 \mathrm{gm}$ at the time of surgery. Experiments were conducted in accordance with the regulations laid down in the United Kingdom Animals (Scientific Procedures) Act, 1986. 
A

\section{1) Sustain nose poke in centre hole for variable delay}

\section{2) Lateralised light stimulus presented}

\section{3) Animals responds in previously lit hole}
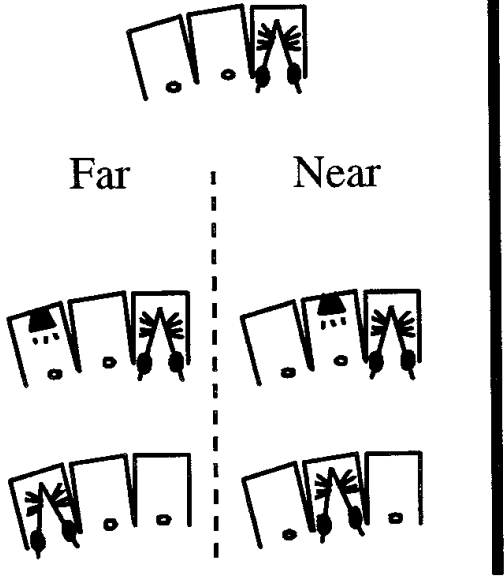

B
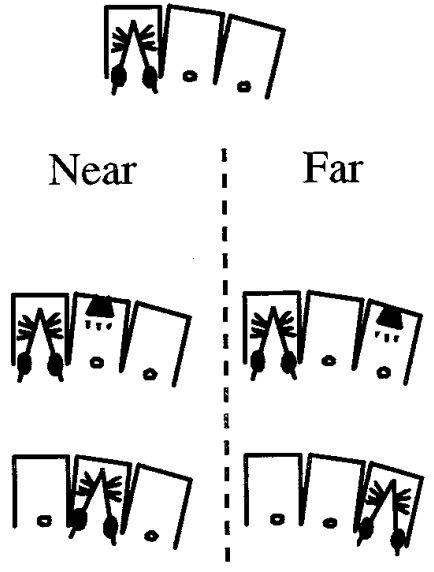

Figure 1. Task requirements when response holes are configured to the left $(A)$ and right $(B)$. The rat must sustain a nose poke in the center hole. After a variable period (delay, 50, 250, 450, or $650 \mathrm{msec}$ ) a brief light flash appeared in one of the two holes to the side of the rat. The rat then had to withdraw its nose from the center and poke its nose into the same hole in which the light had appeared to obtain food reward.

\section{Apparatus}

Testing was conducted in the nine-hole box apparatus (Paul Fray Ltd., Cambridge, UK). Each box measured $26 \times 26 \times 26 \mathrm{~cm}$ with an arc of nine contiguous apertures set into the curved rear wall. Each aperture was $2.5 \mathrm{~cm}$ square and $5 \mathrm{~cm}$ deep. Light-emitting diodes at the rear of each hole could be turned on and off automatically to provide visual cues specific to each hole. Vertical photocell beams at the front of each hole allowed the recording of response latencies and locations. In the opposite panel of the chamber was a food hopper behind a hinged Plexiglas panel to which food pellets ( $45 \mathrm{mg}$; Noyes) could be dispensed. A light in the hopper came on when food was delivered and was extinguished when the food was collected. A house light in the center of the ceiling was capable of illuminating the entire chamber, although the chamber was not illuminated during normal trials. Each chamber was fitted within a soundattenuating box, which in turn was equipped with an extractor fan, which provides a constant low level of background noise. An Acorn A5000 microcomputer running the Arachnid operating system (Paul Fray Ltd.) controlled, and recorded data from, each box independently.

In this study, there were two types of hole configuration, each of which utilized three apertures, with the other holes occluded with metal caps. For the first configuration, only the center hole and the two holes immediately to its left were exposed. For the second configuration, only the center hole and the two holes immediately to its right were open (Fig. 1). A more detailed description of the apparatus can be found elsewhere (Robbins et al., 1993).

\section{Behavioral test procedures}

Training. Subjects were placed on a restricted food regimen, and $24 \mathrm{hr}$ later they were exposed to the operant chamber. This habituation period lasted $30 \mathrm{~min}$, during which animals had access to food pellets that had been placed in the food hopper. All the stimulus holes were capped, and no lights were on during this session. The following day, a training program commenced, whereby only the center hole was exposed. A nose poke in the lit hole extinguished the stimulus light and resulted in a pellet being delivered to the hopper. The light situated in the hopper was lit until the animal entered the hopper. Once the animal had learned to do this, collecting 50 pellets in a $30 \mathrm{~min}$ session, it began the next training program. In this second program, the center hole and two response holes either immediately to the left or the right were exposed. The response holes that were used changed each day. The animal was required to nose poke in the center hole as before. This extinguished the center light and turned on the stimulus light in the response hole. This light remained on until the animal produced a nose poke in the illuminated response hole. The stimulus light was then extinguished, and food was delivered to the hopper as before. Once an animal learned this aspect of the task, it progressed to the complete task paradigm described below. The task parameters of delay, stimulus duration, and maximum response time were introduced at this stage, and these were gradually changed as performance improved.

Task paradigm. The lateralized visual reaction time task is illustrated schematically in Figure 1. Animals were trained to perform the task for each of the two configurations of holes separately, on alternate days, the task for each configuration differing only in the side (left or right) to which it was performed. These two arrangements are referred to as the ipsilateral and contralateral configurations and are named with respect to the side on which an animal was lesioned after training. At the start of each trial only the center light was on. All other lights, including the house lights, were off. The rat was required to poke its nose into the center hole. This extinguished the center light. The rat then had to sustain this nose poke for a variable period (delay) until a brief unpredictable visual stimulus (maximum $0.3 \mathrm{sec}$ ) appeared in one of the two side holes. There were four delays used $(50,250,450$, and $650 \mathrm{msec})$, an equal number of which were presented pseudorandomly during a session. Once the lateralized visual stimulus had occurred, the rat had to withdraw its nose from the center hole (which extinguished the stimulus light if it was still on) and to move and poke its nose into the side hole in which the light had been presented. A correct response was rewarded with the delivery of a food pellet into the food hopper. An incorrect response was punished by the house light being turned on for $1 \mathrm{sec}$ (timeout) and no food being presented. The animal was also punished with a timeout if it withdrew its nose from the center hole before the lateralized stimulus was presented (premature response) or if no response was made to the lateralized stimulus within $5 \mathrm{sec}$ (late response). The next trial was initiated when the animal pushed the panel of the food hopper. A response into the center hole in response to the stimulus presentation was also punished, but there were few such responses under any conditions and are therefore not reported here.

Reaction time was defined and recorded as the latency to initiate the response (i.e., to withdraw the nose from the center hole) after the presentation of the lateralized light stimulus. Movement time was defined and recorded as the latency to produce a nose poke response after the point in time of withdrawal from the center hole. The trial outcome and response location for each trial were also recorded.

Testing procedure. Each session consisted of 80 trials (10 trials at each of the four delays for each response location) and was terminated if not completed after $1 \mathrm{hr}$. Every subject ran two sessions each day, with the response holes on the same side for both sessions. The side of the response holes alternated each day. Animals were judged to have learned the task once their performance, as measured by the percentage of correct trials, had reached an asymptotic level for each side. Data from the 20 sessions, 10 to each side, that were performed before surgery were used for preoperative baseline measures. Animals were then assigned to 
receive lesion or sham surgery, and the groups were matched with respect to the percentage of correct trials achieved. The side of lesion was also determined in this way, with all rats receiving unilateral surgery opposite their most accurate side. Testing resumed $6 \mathrm{~d}$ after surgery for an additional $10 \mathrm{~d}$. Postoperative measures were obtained from the 20 sessions, 10 to each side, that were performed after surgery. An additional two sessions, one to each side, were run about 4 weeks after surgery, to investigate the stability of the lesion deficit over time.

\section{Surgery}

Ten animals received excitotoxic lesions, and eight received identical surgery with PBS. The rats were anesthetized with $0.25-0.3 \mathrm{ml} / \mathrm{kg}$ Hypnorm injected intramuscularly and $0.25 \mathrm{ml}$ of Diazepam injected intraperitoneally and were placed in a stereotaxic frame. Unilateral striatal lesions were given by injecting $2 \times 0.5 \mu \mathrm{l}$ of $0.09 \mathrm{M}$ quinolinic acid into two sites in the striatum. The toxin was delivered by a 30 gauge cannula, attached by polyethylene tubing to a $10 \mu$ l glass syringe, which was mounted on a microdrive pump. Coordinates were anterior (A), 0.0 $\mathrm{mm}$; lateral $(\mathrm{L}), 3.6 \mathrm{~mm}$; and ventral $(\mathrm{V}),-4.5 \mathrm{~mm}$; and A, $1.2 \mathrm{~mm}$; $\mathrm{L}$, $2.8 \mathrm{~mm}$; and $\mathrm{V},-4.5 \mathrm{~mm}$, with the nose bar set at $-2.3 \mathrm{~mm}$ (Paxinos and Watson, 1986). Measurements were taken anterior to bregma, lateral to the midline, and dorsal to dura. Each infusion lasted for $4 \mathrm{~min}$, with an additional $5 \mathrm{~min}$ allowed for diffusion before the cannula being withdrawn. One animal in the lesion group died postoperatively.

\section{Postoperative challenges}

Extinction task and no stimuli condition. About 1 month postoperatively, animals were tested with probe trials, which examined the effect of presenting both stimuli simultaneously, a test that can be considered an analog to the "extinction" task used in assessing neglect patients clinically (Bender, 1952; Valenstein and Heilman, 1981). Trials in which no stimuli were presented were also given. Animals were tested for one session on each of the two response hole configurations on separate days. Each session consisted of 80 trials and comprised three interspersed trial types: (1) control trials, in which only one stimulus light was presented; (2) trials in which both stimulus lights were presented; and (3) trials in which no stimulus lights were presented. There were 40 control trials, and 20 trials each in the other two conditions. When both stimuli were presented, a response in either hole was rewarded. When neither stimulus was presented, a delay period of $0 \mathrm{msec}$ was used, and a response in either hole was punished. The primary dependent variable of interest for these challenges was the near hole response bias.

Bilaterally configured response options. To assess performance when the response holes were not adjacent, animals were also exposed postoperatively to four other hole configurations, in addition to the ipsilateral and contralateral configurations, which have already been described. Each configuration still consisted of a center hole and two of the previously used response holes, with animals still being appetitively reinforced for responding in a hole where a light occurred. These additional response hole arrangements were termed as follows: near configuration (near ipsilateral and near contralateral holes); far configuration (far ipsilateral and far contralateral holes); near ipsilateral/far contralateral configuration; and near contralateral/far ipsilateral configuration. Animals performed one session for each of these bilaterally configured response options, with the extinction task described above used on each occasion.

These bilateral configurations allowed for the testing of two hypotheses. First, it was possible to examine further the concept of egocentric response coding. Two of the configurations (near ipsilateral/far contralateral configuration and near contralateral/far ipsilateral) maintain the same distance between the response holes but vary their position relative to the center hole and thus the body axis. A difference in bias in these two conditions would be expected only if response space is egocentrically coded. Second, responding in near and far space could be examined separately using the "near/near" and "far/far" configurations. This allowed for the analysis of a potential dissociation between near and far space by assessing whether the lesion produced similar deficits in the two conditions, particularly when competition between the two responses was greatest in the extinction condition.

\section{Behavioral measures}

The following behavioral measures were subjected to analysis: (1) Accuracy (correct responses as a percentage of correct and incorrect trials). (2) Response bias; for the ipsilateral and contralateral configurations, a near hole bias was calculated. This was expressed as the number of correct and incorrect responses made to the near hole as a percentage of the total number of correct and incorrect responses made to either hole. Thus, a bias score of $50 \%$ represents no bias, $>50 \%$ denotes a bias toward the near hole, and $<50 \%$ denotes a bias in responding toward the far hole. For the four hole configurations that are introduced postoperatively, an ipsilateral bias score is calculated in a similar manner. Therefore a bias score of $>50 \%$ denotes an ipsilateral bias, and $<50 \%$ denotes a contralateral bias. (3) Premature responses (as a percentage of all attempted trials). (4) Late responses (as a percentage of all attempted trials). (5) Reaction time for correct responses. (6) Movement time for correct responses.

\section{Treatment of data}

Data were collected automatically for every trial, for each box. Data for each animal were then collated over all relevant sessions preoperatively and postoperatively. Based on an exploratory analysis of the reaction time distribution, a reaction time limit of $>70 \mathrm{msec}$ was imposed when processing the data; trials with reaction times of $<70 \mathrm{msec}$ were classified as premature responses. When averaging latencies, a geometric mean was calculated for each rat for each treatment combination. This transformation results in outlying data points having less influence than they do on an arithmetic mean. Overall means of reaction and movement times represent the arithmetic average of the geometric means of individual rats.

The reaction and movement time data collected for the ipsilateral and contralateral configurations were subject to a repeated measures ANOVA with the five factors: group, surgery, side, delay, and distance (near or far). The percentages of premature and late responses were analyzed in a similar manner but without the factor of delay. The bias data were transformed with an arc sin function, and the transformed scores were analyzed with a repeated measures ANOVA using the factors surgery, side, and group. The Genstat 5 for Windows (release 3.2) statistical package (Experimental Research Station, Rothampsted, UK) was used for all statistical computations. Post hoc tests were based on Sidak's comparisons (Rohlf and Jokal, 1995) as appropriate.

\section{Histological analysis}

Once behavioral testing had been completed, the rats were anesthetized with $1 \mathrm{ml}$ of Euthatal and were transcardially perfused with $100 \mathrm{ml}$ of PBS, followed by $250 \mathrm{ml}$ of $10 \%$ formalin. The brains were removed, post-fixed in $10 \%$ formalin for $24 \mathrm{hr}$, and then removed in $30 \%$ sucrose in PBS until they sank. The tissue was sectioned serially at $60 \mu \mathrm{m}$ on a freezing microtome. Two parallel 1:6 series were mounted on slides. One series was stained with cresyl violet, and the other for acetylcholinesterase activity.

A SeeScan (Cambridge, UK) image analysis system was used to quantify striatal and ventricular volumes for both the lesion and intact side. The histological measures of striatal volume and ventricular volume were calculated as a percentage of the volume for the intact side for each animal. Sections were photographed digitally, and Adobe Photoshop 3.0 for Windows was used in preparing photomicrographs.

\section{RESULTS}

\section{Histological analysis}

Infusions of quinolinic acid resulted in discrete lesions of the dorsal striatum. The lesioned area was distinguished by profound neuronal loss within the dorsal striatum, a reduction in striatal volume, and a corresponding increase in size of the lateral ventricles. The acetylcholinesterase stain showed a reduction in enzyme activity for areas that coincided to areas of cell loss shown by the Nissl stain (Fig. 2). There was no evidence of damage to adjacent structures such as the globus pallidus, nor did cell loss extended to the ventral striatum. There was slight cortical damage in one animal in the lesion group.

The volume of the lesioned striatum, as a percentage of an animal's intact striatum, was $76.1 \%$ for animals that received injections of quinolinic acid. This reduction was not seen for sham-lesioned animals $(107.1 \%)$, as indicated by an independent $t$ test $(t=3.04 ; p<0.05)$. There was a corresponding increase in ventricular volume on the lesioned side for excitotoxically lesioned animals when compared with the sham-operated group (lesion, 183.3\%; sham, 85.4\%; $t=4.57 ; p<0.01$ ). 


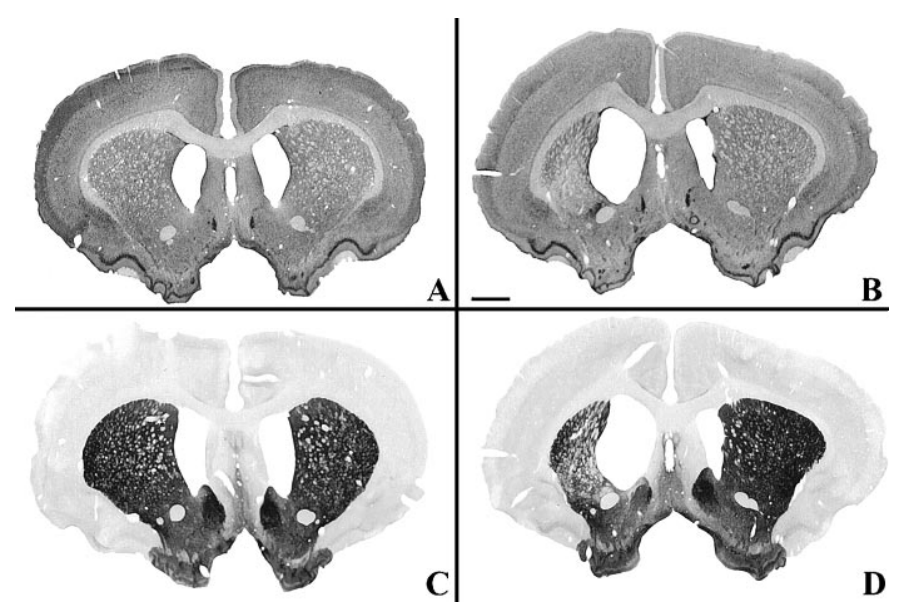

Figure 2. Photomicrographs illustrating the effects of unilateral injections into the dorsal striatum of buffer solution $(A, C)$ and $0.09 \mathrm{M}$ quinolinic acid $(B, D)$. The lesion produced extensive neuronal loss, as shown by the Nissl stain $(B)$. The acetylcholinesterase-stained section exhibits a marked decrease in enzyme activity over a similar area $(D)$. Scale bar, $1 \mathrm{~mm}$.

\section{Accuracy}

Preoperatively, the accuracy of the two groups was $77.6 \%$ (lesion) and $78.1 \%$ (sham). After surgery the lesion group responded less accurately $(58.3 \%)$, but the sham group (77.2\%) did not. (group $\times$ surgery, $\left.F_{(1,15)}=22.89 ; p<0.01\right)$. This decline in accuracy for the lesion group was not lateralized (group $\times$ surgery $\times$ sides, $F(1,15)=2.49$; NS), but lesioned animals tended to be less accurate on the contralateral side (ipsilateral, 63.5\%; contralateral, 53.0). However, this inaccuracy is associated with, and probably determined by, large changes in response bias.

\section{Bias}

Preoperative and postoperative bias scores are shown in Figure 3. Before surgery, all rats showed a slight near hole response bias (overall untransformed bias score, 58.1\%). Postoperatively, lesioned rats directed virtually all contralateral responses toward the near hole (untransformed bias score, 99.3\%). There was a less marked, nonsignificant, increase in bias shown by lesioned rats when responding toward the ipsilateral side hole (untransformed bias score, $74.8 \%$ ), as revealed by a significant three-way interaction between group, surgery, and side $\left(F_{(1,15)}=13.29 ; p<0.01\right)$. Sham animals had bias scores of $57.7 \%$ (ipsilateral) and $60.7 \%$ (contralateral) after surgery.

\section{Premature responses}

The percentages of premature responses made preoperatively were $19.38 \%$ (lesion) and $20.68 \%$ (sham). Postoperatively, more premature responses were produced by lesion group (33.3\%) than by sham animals $(21.61 \%)\left(\right.$ group $\times$ surgery, $F_{(1,15)}=18.11 ; p<$ 0.01 ). This effect was not specific to either side (group $\times$ surgery $\times$ side, $\left.F_{(1,15)}=1.41 ; \mathrm{NS}\right)$, the lesion group responding prematurely $31.51 \%$ (ipsilaterally) and $35.17 \%$ (contralaterally) in postoperative sessions.

\section{Late responses}

There were few late responses before surgery (lesion, $0.14 \%$; sham, $0.45 \%$ ). Lesioned animals produced more late responses after surgery (lesion, $1.12 \%$; sham, $0.16 \%$; group $\times$ surgery, $\left.F_{(1,15)}=20.76 ; p<0.001\right)$, and this tended to be the case specifically for responding on the contralateral side (group $\times$

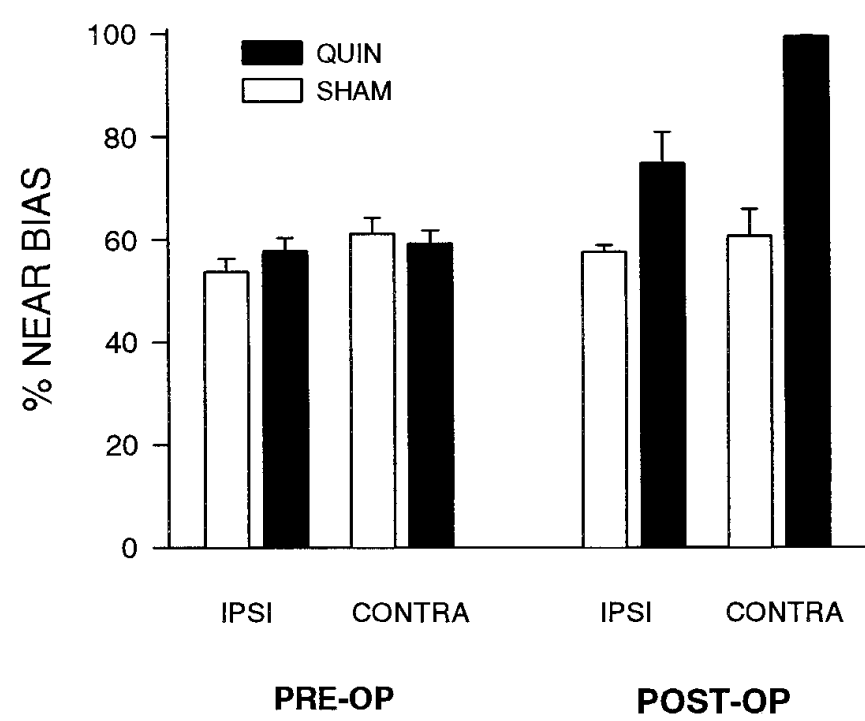

Figure 3. Response bias to the near hole, for both groups when performing the task to the side ipsilateral (IPSI) or contralateral $(C O N T R A)$ to the lesion $[\% N E A R B I A S=$ (all correct and incorrect responses to the near hole)/(correct and incorrect responses to both holes)]. Before the lesion, both groups displayed a mild bias toward the near hole. Postoperatively, lesioned animals directed virtually all contralateral responses toward the nearer of the two holes. They also showed a less marked increase in near hole bias on the ipsilateral side. Bars indicate the SEM of individual animal means.

surgery $\times$ side, $\left.F_{(1,15)}=6.66 ; p<0.05\right)$. However, there were very few responses of this kind (the average number of such responses never exceeded $2 \%$ per session, for any condition).

\section{Reaction time}

Figure 4 shows the reaction time performance, for correct trials only, of both groups before and after surgery. There were no differences between the groups preoperatively, with reaction times decreasing as a function of increasing delay period for all animals (delay, $F_{(3,45)}=19.72 ; p<0.001$ ). There was no difference in the time taken to initiate responses to either hole on either side.

The lesion had no effect on the reaction times for responses made toward the ipsilateral side. However, the lesioned group did show an increase in reaction times for contralateral responses. $\left(\right.$ group $\times$ surgery $\times$ side, $\left.F_{(1,15)}=6.16 ; p<0.05\right)$. Lesioned animals were, on average, $60 \mathrm{msec}$ slower to initiate responses to the contralateral near hole (sham, 187; lesion, $247 \mathrm{msec}$ ). The lesion group still showed a speeding of reaction time as the delay period increased, although the four-way interaction of group $\times$ surgery $\times$ side $\times$ delay suggested that this contralateral increase in lesioned animals was delay-dependent $\left(F_{(3,45)}=3.35 ; p<\right.$ $0.05)$. Lesioned animals did not complete sufficient trials to the contralateral far hole to enable a full factorial analysis of reaction times.

\section{Movement time}

Figure 5 shows the movement time performance for both groups before and after surgery. There was no difference between the groups preoperatively in the time taken to complete a response. Latencies were consistently greater for responses to the far holes (distance, $F_{(1,15)}=196.29 ; p<0.001$ ), and the delay period had no influence on movement times.

After surgery, there were no lateralized impairments in le- 


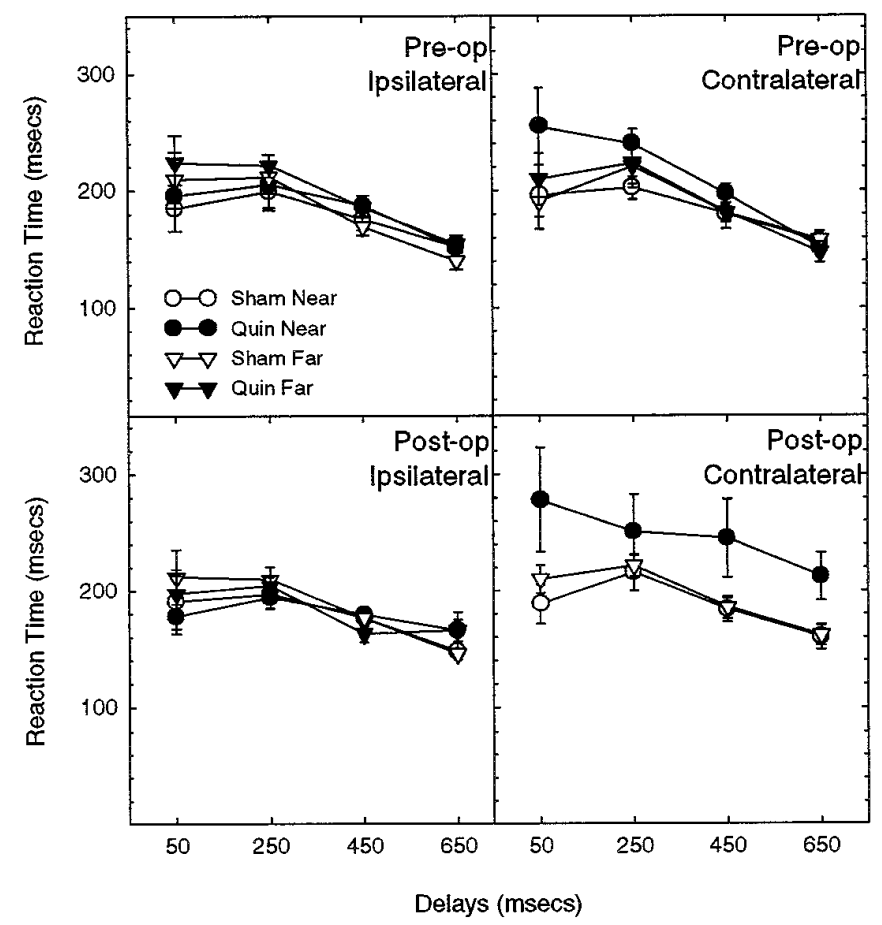

Figure 4. Preoperative and postoperative reaction times for correct responses, plotted as a function of delay, for each group. The lesion group showed no postoperative increase in reaction time when responding ipsilaterally. However, it did show an increase in initiating responses, which were contralateral to the side of the lesion, at each delay.

sioned animals (group $\times$ surgery $\times$ side; $F_{(1,15)}<1.00 ; \mathrm{NS}$ ). However, the lesion group was marginally slower to complete a response to the far hole, although on the ipsilateral side (group $\times$ surgery $\times$ distance, $\left.F_{(1,15)}=7.45 ; p<0.05\right)$.

\section{Effect of presenting neither or both stimulus lights (extinction) for ipsilateral and contralateral hole configurations}

As shown in Figure 6, neither the extinction test nor the absence of stimulus lights affected the response bias of the lesion or sham animals when the response holes were presented unilaterally (stimulus, $F_{(2,30)}=1.49$; NS).

Ipsilaterally, the stimulus condition had little effect on either the lesion or sham group. Neither group showed an increase in response bias when both ipsilateral holes were lit, and both showed only a slight increase in bias toward the near hole when no light was presented.

Contralaterally, the lesioned animals continued to show a strong near hole bias when responding (group $\times$ holes, $F_{(1,15)}=$ 30.99; $p<0.01$ ) and did so regardless of whether either, both, or none of the response holes were cued (group $\times$ holes $\times$ stimulus, $\left.F_{(2,30)}=0.89 ; \mathrm{NS}\right)$.

\section{Effect of bilateral response hole configurations}

When the bilateral response hole configurations were used, animals displayed a significantly greater bias toward the ipsilateral hole for two of the configurations: far ipsilateral/far contralateral and near ipsilateral/far contralateral (hole, $F_{(3,45)}=5.16 ; p<$ $0.01)$. This was marginally affected by the dorsal striatal lesion, the interaction between group and hole bordering on significance $\left(F_{(3,45)}=2.66 ; p=0.059\right)$. Figure 7 illustrates how lesioned rats

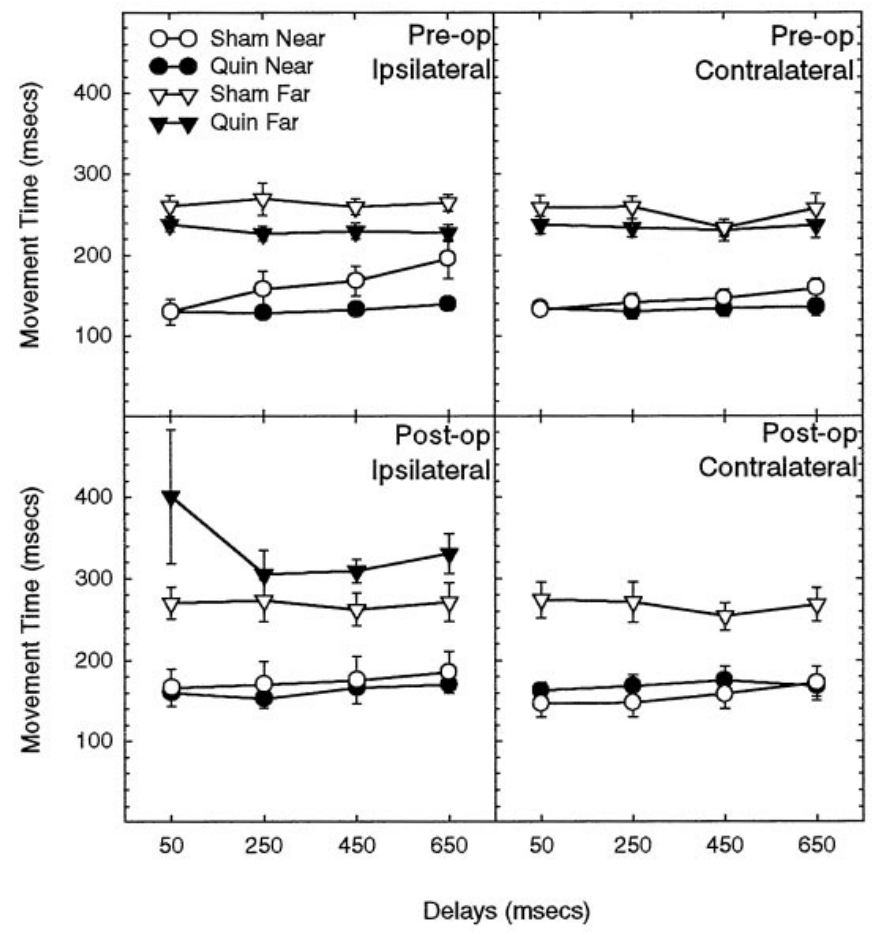

Figure 5. Preoperative and postoperative movement times for correct responses, plotted as a function of delay, for each group. The lesion group showed no lateralized impairments after surgery, although it took slightly longer to complete far hole responses.

biased their responding toward the ipsilateral side in these two conditions, both of which include the far contralateral hole.

The extinction test brought out significant differences in performance between the two groups in these bilateral response hole configurations (stimulus $\times$ group, $F_{(2,30)}=4.07 ; p<0.05$ ). The effect of presenting both stimuli was to increase the bias toward the ipsilateral hole in the lesion group compared with control animals (Sidak $\left.t_{(3,30)}=3.63 ; p<0.01\right)$. When no response was cued, lesioned animals showed no increase in response bias and were no different than control animals in this regard (Sidak $t_{(3,30)}$ $=0.13$; NS).

\section{Lack of recovery for response bias}

Response bias was analyzed for contralateral sessions, which were run $6-7 \mathrm{~d}$ and 4 weeks after surgery, to see whether there was any reduction in the behavioral deficit over time. Bias scores in lesioned animals were unchanged over time (time $\times$ group, $F_{(1,15)}$ $=1.74 ; \mathrm{NS})$. The untransformed bias scores for lesioned animals were $92.6 \%$ and $93.7 \%$ for the premature and late sessions, respectively.

\section{Correct versus incorrect reaction times for lesioned animals}

To investigate whether lesioned animals were able to discriminate between the two stimuli in the visual field contralateral to the lesion, correct and incorrect reaction times for responses to the near hole were examined and compared with the pattern seen ipsilaterally in the lesion group. On the ipsilateral side, correct responses to the near hole were initiated faster than incorrect responses to the near hole, at all delays. Despite the overall slowing of reaction times for contralateral responses, this differ- 

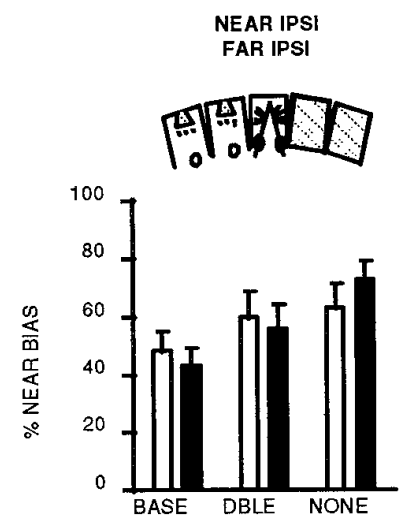
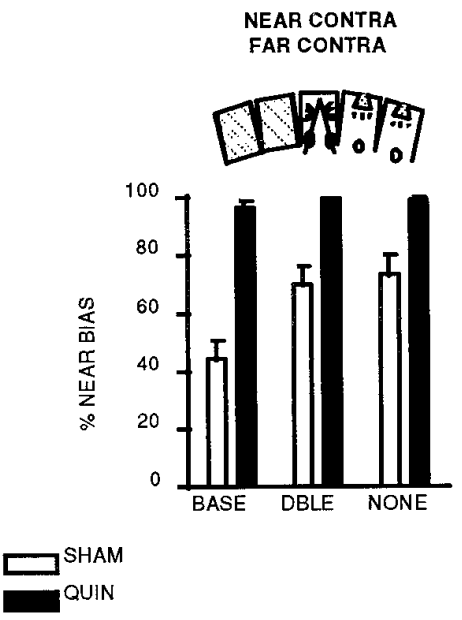

Figure 6. Near hole response bias for ipsilateral (IPSI) and contralateral (CONTRA) configurations for postoperative sessions when trials consisting of both stimuli (extinction) and no stimuli were presented. On the contralateral side, lesioned animals still responded virtually exclusively to the far hole in all stimulus conditions. Ipsilaterally, the lesion group performed as shams. $B A S E$, Either light; $D B L E$, both lights; NONE, no lights presented. The hole configurations are shown above each graph, as they would appear for an animal lesioned on the left.

ence was also seen on the contralateral side (trial outcome $\times$ side, $F_{(1,8)}=2.56$; NS).

\section{DISCUSSION}

This study has used a novel paradigm for examining a long-lasting form of neglect in the rat after dorsal striatal damage, which helps us understand the spatial nature of the neglect and the normal mode of functioning of the intact striatum.

It is unlikely for a number of reasons that the contralateral deficits seen in this study are primarily sensory in nature. Previous studies that dissociated stimulus and response locations demonstrated that unilateral striatal lesions specifically produce a response-related deficit (Mayer et al., 1992). In addition, there are several lines of evidence in the present study that suggest that lesioned animals are processing many aspects of visual information virtually normally.

First, when rats responded contralaterally, they did so in a delay-dependent manner (Fig. 4). If the nose withdrawal was determined by waiting for a set time, rather than by stimulus presentation, then the reaction times at each delay would differ by the difference in delay periods (i.e., reaction times at the first and last delay would differ by $600 \mathrm{msec}$ ), which clearly they did not. This suggests that lesioned animals were under a degree of stimulus control, even when responding contralaterally. Second, although lesioned animals, when responding contralaterally, did so almost exclusively to the near hole, it appears that the rats could still discriminate between the near and far stimuli. This is suggested by lesioned animals initiating correct responses more quickly than incorrect responses, reflecting the pattern seen ipsilaterally. Furthermore, a sensory attentional deficit would not account for contralateral stimuli eliciting ipsilateral (and therefore incorrect) responses, which are produced for bilateral hole configurations. The contralateral deficit, therefore, would seem to be an impairment in the control of responding to contralateral space. Nor can it be argued that the near hole bias exhibited by the lesioned rats when responding contralaterally is attributable to a simple motor incapacity to move to the far hole, because

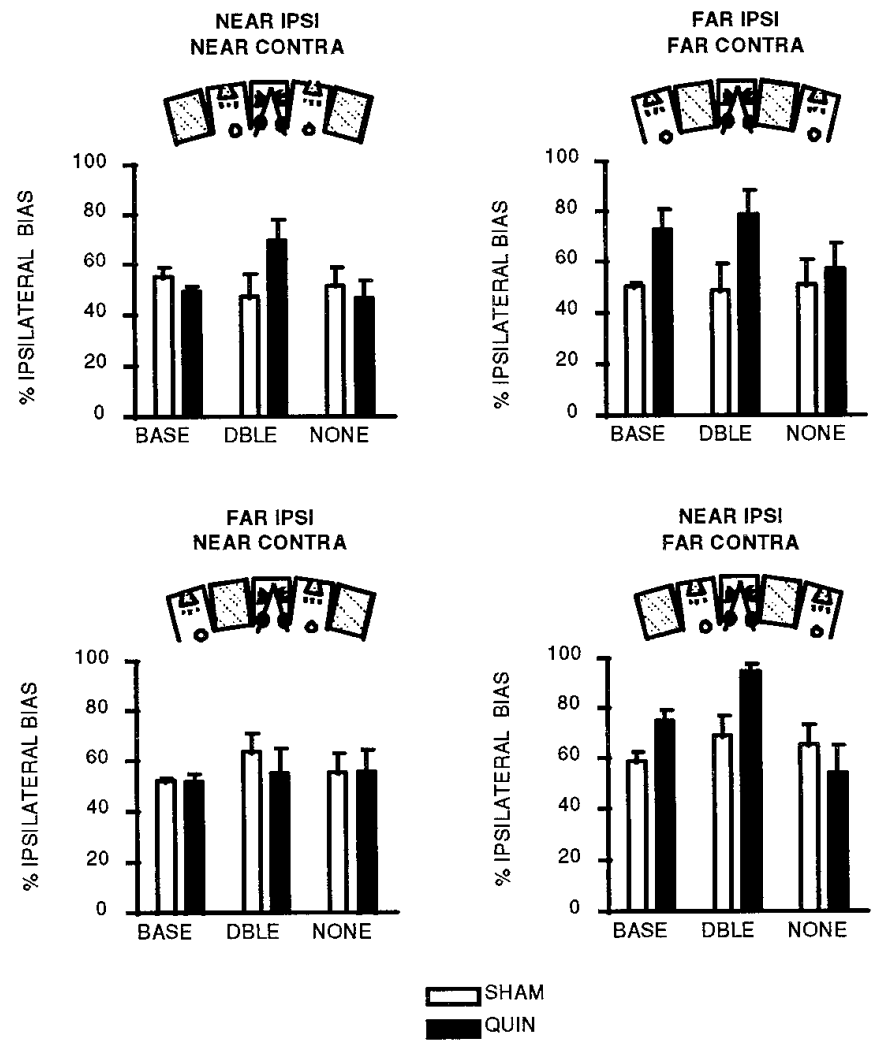

Figure 7. Ipsilateral response bias for the four bilateral hole configurations, for postoperative sessions when trials consisting of both stimuli (extinction) and no stimuli were presented. Lesioned animals tended to bias their responding to the ipsilateral (IPSI) side when the far contralateral $(C O N T R A)$ hole was one of the holes used. The extinction task also tended to increase the ipsilateral bias in lesioned animals. However, lesioned animals showed no bias in those conditions in which no stimulus was presented. $B A S E$, Either light; $D B L E$, both lights; $N O N E$, no lights presented [\% IPSILATERAL BIAS = (all correct and incorrect responses to the ipsilateral hole)/(correct and incorrect responses to both holes)]. The hole configurations are shown above each graph, as they would appear for an animal lesioned on the left.

lesioned animals could move to the far hole during postoperative sessions that involved a bilateral configuration of response holes (Fig. 7). The fact that lesioned animals do not respond in the far hole when both response options are contralateral indicates that this bias is attributable in part to the presence of the near hole and presumably results from competing tendencies to respond there, which are so potent that they occlude responding in the far contralateral hole altogether.

Apart from this clear neglect of contralateral space, there were also secondary, unlateralized deficits. First, premature responding was increased equally on both sides. Second, near hole bias increased slightly on the ipsilateral side, although reaction times to the ipsilateral holes were not affected by the lesion. This is unlikely to be attributable to any disruption of response coding, for reasons that are discussed below. In addition, movement time was retarded mildly to the far ipsilateral hole. Such impairments, however, seem less marked and may reflect general effects of surgery. Thus, the ipsilateral near hole bias was no longer seen in the challenges presented 4 weeks postoperatively, suggesting a degree of recovery, whereas the contralateral bias never recovered (Fig. 6). 


\section{Egocentric versus allocentric response coding}

The evidence discussed above shows that contralateral neglect is not a simple visual or motor deficit, but may reflect how responding is organized spatially. The paradigm used further enabled an examination of the nature of the striatal spatial processing deficit in egocentric or allocentric terms. The logic followed that used to investigate analogous deficits seen in rats with unilateral striatal dopamine depletion (Brown and Robbins 1989a). In the present study, in which animals were trained to perform the task to both sides, lesioned animals were impaired on the contralateral side only. The absence of ipsilateral deficits suggested an egocentric coding of response space. If response space was coded relative to an external referent, such as the response holes themselves, then a bias might be expected toward the relatively ipsilateral hole (i.e., the far hole when the task is performed to the ipsilateral side). This is evidently not the case, even when both response holes are lit (Fig. 6). Therefore, the near hole bias seen for contralateral responding would appear to be the product of a deficit in response space that is defined egocentrically, i.e., with respect to the animal's midline. Such an interpretation is in keeping with the disruption in orienting behavior that accompanies unilateral striatal dopamine depletion (Fairley and Marshall, 1986).

It is possible that the near hole bias seen for contralateral holes may not reflect a disruption of response space but arises instead from an inability to inhibit responses directed toward the near hole. Such a possibility was investigated using the four novel response hole configurations, each of which consisted of ipsilateral and contralateral response apertures. In these circumstances, the lesioned animals direct significantly more responses to the contralateral far hole, suggesting a competitive influence of the near hole when it was available for responding. However, the data are still compatible with an explanation of the deficit in terms of response space. When bilateral configurations of response holes were used postoperatively (Fig. 7), the response bias was more sensitive to the position of the contralateral, rather than the ipsilateral, hole. This is in keeping with the idea of egocentric space coding, which would suggest that ipsilateral responses here were not mediated by the lesioned striatum. In addition, one can also compare the two asymmetric hole configurations, near contralateral/far ipsilateral and near ipsilateral/far contralateral. These two configurations consist of response apertures that are the same distance apart, but the latter is set further to the contralateral side relative to the animal's midline. These two conditions would elicit similar response biases if responding was allocentrically coded. However, the patterns of responding are noticeably different (Fig. 7), with the more contralaterally set holes (near ipsilateral/far contralateral) producing a greater ipsilateral bias in lesioned animals. This is in accordance with bilateral electrolytic lesion studies (Cook and Kesner, 1988; Kesner et al., 1993), electrophysiological data (Wiener, 1993), and clinical evidence (Potegal, 1971), which suggest that that response vectors, as mediated by the striatum, are determined with respect to egocentric coordinates.

\section{Striatal neglect may reflect distinct spatial domains}

The issue of what mediates responding to the near contralateral hole raises three possibilities. First, such responding may not involve the striatum but a separate mechanism unaffected by the lesion. Second, it may be that although response space is organized egocentrically, each hemispace governed by the two striata overlaps at the midline, and it is therefore the intact striatum that initiates responding to the near contralateral hole. However, neither of these possibilities would account for the increase in correct reaction times for responses to the contralateral near hole (Fig. 4). The third option is that contralateral responses were mediated by residual striatum on the lesioned side. The bias seen contralaterally could therefore reflect a collapse toward proximal space, as is the case after parietal damage (Ladavas, 1987). However, converging anatomical data suggest that the striatum is organized in a somatotopic, rather than a spatial, manner (Alexander and Crutcher, 1990) (for review, see Parent and Hazrati, 1995). This raises the possibility that dorsal striatal lesions impact on distinct spatial domains. Rizzolatti et al. (1983) showed that discrete lesions of the frontal eye field and inferior premotor cortices in primates resulted in deficits in far (extrapersonal) space and near (peripersonal) space, respectively. This dissociation may provide a context in which to interpret the findings of the current study.

The effect of the lesion is clarified when the lesioned striatum is put in competition with the intact striatum, as in the bilateral hole configurations. The enhanced bias seen when both stimuli are presented simultaneously may reflect the outcome of competition between processing in the intact and lesioned striatum, each of which would normally exhibit a degree of independence. Thus, competition normally occurs between striata as well as between response sets for different responses within each striatum. When the striatum is lesioned unilaterally there are thus two effects: bias to ipsilateral space and bias to the proximal portion of contralateral space. It is possible that competition may only occur between striata for near peripersonal space. This would readily account for the unexpected finding that there was no significant extinction effect was observed in the far ipsilateral/far contralateral condition. The deficit in responding to the distal location contralaterally may represent a specific impairment in responding to locations in far extrapersonal space. This is in keeping with the separate mechanisms that have been shown to exist in the frontal cortices of the primate for distinct spatial domains (Rizzolatti et al., 1983), and furthermore, the neural structure responsible (frontal eye field) converges with parietal input to the striatum. The abolition of bias with no visual stimulus, and the lack of lateralized premature responses, suggests that it arises from an interaction between an internal disposition and the presence of specific cues for instrumental action.

\section{REFERENCES}

Alexander GE, Crutcher MD (1990) Functional architecture of basal ganglia circuits: neural substrates of parallel processing. Trends Neurosci 13:266-271.

Bender MB (1952) Disorders of perception. Springfield, IL: Thomas.

Bisiach E, Luzzatti C (1978) Unilateral neglect of representational space. Cortex 14:129-133.

Bisiach E, Luzzatti C, Perani D (1979) Unilateral neglect, representational schema and consciousness. Brain 102:609-618.

Bisiach E, Perani D, Vallar G, Berti A (1986) Unilateral neglect: personal and extrapersonal. Neuropsychologia 24:759-767.

Brown VJ, Robbins TW (1989a) Deficits in response space following unilateral striatal dopamine depletion in the rat. J Neurosci 9:983-989.

Brown VJ, Robbins TW (1989b) Elementary processes of response selection mediated by distinct regions of the striatum. J Neurosci 9:3760-3765.

Caramazza A, Hillis AE (1990) Spatial representation of words in the brain implied by studies of a unilateral neglect patient. Nature 346:267-269.

Carli M, Evenden JL, Robbins TW (1985) Depletion of unilateral striatal dopamine impairs initiation of contralateral actions and not sensory attention. Nature 313:679-682.

Carli M, Jones GH, Robbins TW (1989) Effects of unilateral dorsal and 
ventral striatal dopamine depletion on visual neglect in the rat: a neural and behavioural analysis. Neuroscience 29:309-327.

Cook D, Kesner RP (1988) Caudate-nucleus and memory for egocentric localization. Behav Neural Biol 49:332-343.

Cowey A, Small M, Ellis S (1994) Left visuospatial neglect can be worse in far than in near space. Neuropsychologia 32:1059-1066.

Driver J, Halligan P (1991) Can visual neglect operate in object centred coordinates? An affirmative single-case study. Cognit Neuropsychol 8:475-496.

Dunnett SB, Björklund A (1983) Conditioned turning in rats: dopaminergic involvement in the initiation of movement rather than the movement itself. Neurosci Lett 41:173-178.

Fairley PC, Marshall, JF (1986) Dopamine in the lateral caudateputamen of the rat is essential for somatosensory orientation. Behav Neurosci 100:652-663.

Farah MJ, Brunn JL, Wong AB, Wallace MA, Carpenter PA (1990) Frames of reference for allocating attention to space-evidence from the neglect syndrome. Neuropsychologia 28:335-347.

Halligan PW, Marshall JC (1991) Left neglect for near but not far space in man. Nature 350:498-500.

Harper P (1996) Huntington's disease, Ed 2. London: Saunders.

Heilman KM, Bowers D, Coslett HB, Whelan H, Watson RT (1985) Directional hypokinesia-prolonged reaction-times for leftward movements in patients with right hemisphere lesions and neglect. Neurology 35:855-859.

Karnath HO, Schenkel P, Fischer B (1991) Trunk orientation as the determining factor of the contralateral deficit in the neglect syndrome and as the physical anchor of the internal representation of body orientation in space. Brain 114:1997-2014.

Kesner RP, Bolland BL, Dakis M (1993) Memory for spatial locations, motor-responses, and objects-triple dissociation among the hippocampus, caudate-nucleus, and extrastriate visual-cortex. Exp Brain Res 93:462-470.

Ladavas E (1987) Is the hemispatial deficit produced by right parietal lobe damage associated with retinal or gravitational coordinates? Brain 110:167-180.
Mayer E, Brown VJ, Dunnett SB, Robbins TW (1992) Striatal graftassociated recovery of a lesion-induced performance deficit in the rat requires learning to use the transplant. Eur J Neurosci 4:119-126.

Mittleman G, Brown VJ, Robbins TW (1988) Intentional neglect following unilateral ibotenic acid lesions of the striatum. Neurosci Res Comm 2:1-8.

Parent A, Hazrati L-N (1995) Functional anatomy of the basal ganglia. I. The cortico-basal ganglia-thalamo-cortical loop. Brain Res Rev 20:91-127.

Paxinos G, Watson C (1986) The rat brain in stereotaxic coordinates. North Ryde, Australia: Academic.

Posner MI, Walker JA, Friedrich FJ, Rafal RD (1984) Effects of parietal injury on covert orienting of attention. J Neurosci 4:1863-1874.

Potegal M (1971) A note on spatial-motor deficits in patients with Huntington's disease: a test of a hypothesis. Neuropsychologia 9:233-235.

Rizzolatti G, Matelli M, Pavesi G (1983) Deficits in attention and movement following the removal of postarcuate (area 6) and prearcuate (area 8) cortex in macaque monkeys. Brain 106:655-673.

Robbins TW, Muir JL, Killcross AS, Pretsell D (1993) Methods of assessing attention and stimulus control in the rat. In: Behavioural neuroscience, Vol I (Sahgal A, ed), pp 13-47. Oxford: IRL.

Rohlf FJ, Jokal RR (1995) Statistical tables, Ed 3. New York: Freeman. Shelton PA, Bowers D, Heilman KM (1990) Peripersonal and vertical neglect. Brain 113:191-205.

Tegner R, Levander M (1991) Through a looking glass: a new technique to demonstrate directional hypokinesia in unilateral neglect. Brain 114:1943-1951.

Valenstein E, Heilman KM (1981) Unilateral hypokinesia and motor extinction. Neurology 31:445-448.

Watson RT, Miller BD, Heilman KM (1978) Nonsensory neglect. Ann Neurol 3:505-508.

Wiener SI (1993) Spatial and behavioral correlates of striatal neurons in rats performing a self-initiated navigation task. J Neurosci 13:38023817. 